\title{
Semi-nested polymerase chain reaction-based detection of Babesia spp. in small ruminants from Northwest of Iran
}

\author{
Ahad Bazmani ${ }^{1}$, Amir Abolhooshyar ${ }^{2}$, Abbas Imani-Baran ${ }^{3}$ and Hamid Akbari ${ }^{4}$
}

1. Infectious and Tropical Diseases Research Center, Tabriz University of Medical Sciences, Tabriz, Iran; 2. Department of Pathobiology, Faculty of Veterinary Medicine, University of Tabriz, Tabriz, Iran; 3. Department of Pathobiology, Faculty of Veterinary Medicine, University of Tabriz, Tabriz, Iran; 4. Department of Clinical Sciences, Faculty of Veterinary Medicine, University of Tabriz, Tabriz, Iran.

Corresponding author: Abbas Imani-Baran, e-mail: a.imani@tabrizu.ac.ir

Co-authors: AB: bazmany_ahad@yahoo.com, AA: hoshyarvet81@gmail.com, HA: h.akbari@tabrizu.ac.ir Received: 02-10-2017, Accepted: 24-01-2018, Published online: 03-03-2018

doi: 10.14202/vetworld.2018.268-273 How to cite this article: Bazmani A, Abolhooshyar A, Imani-Baran A, Akbari H (2018) Semi-nested polymerase chain reaction-based detection of Babesia spp. in small ruminants from Northwest of Iran, Veterinary World, 11(3): 268-273.

\begin{abstract}
Aim: The present study aimed to detect Babesia ovis and Babesia motasi in the blood samples of sheep and goats from Northwest of Iran by the semi-nested polymerase chain reaction (PCR) technique.

Materials and Methods: A total of 166 whole blood samples (including 123 sheep and 43 goats) were collected. In the first stage, the PCR was performed to amplify a piece of $18 \mathrm{~S}$ rRNA gene of Babesia and Theileria genera. Then, semi-nested PCR was carried out on all PCR products to differentiate B. ovis and B. motasi.
\end{abstract}

Results: The PCR indicated that totally, 19 (11.44\%) out of 166 samples were positive for Babesia or Theileria spp. The semi-nested PCR showed that 38 samples $(22.89 \%)$ were positive only for B. ovis. No significant association was found between the infection rate of $B$. ovis and age, gender and species of animals.

Conclusion: In the present study, there was no evidence for B. motasi infection in small ruminants from Northwest of Iran. Therefore, B. ovis was the main causative agent of ovine Babesiosis in this region.

Keywords: Babesia motasi, Babesia ovis, Iran, semi-nested polymerase chain reaction, small ruminants.

\section{Introduction}

In small ruminants, babesiosis is associated with intraerythrocytic protozoan parasites such as Babesia ovis, Babesia motasi, and Babesia crassa which is characterized more commonly by fever, anemia, hemoglobinuria, and icterus [1,2]. The most pathogenic species of Babesia in small ruminants is B. ovis. However, $B$. motasi has a moderate virulence [3]. Ixodid ticks including Rhipicephalus and Haemaphysalis are the main vector of $B$. ovis and B. motasi, respectively [2]. Ovine babesiosis is of considerable economic importance in tropical and subtropical areas [4]. Therefore, screening and control programs should be considered to reduce the negative impact of the disease on livestock farming. The identification of Babesia spp. is commonly performed by microscopic examination of Giemsa stained blood smears. However, its low sensitivity has limited the utilization of this method in the epidemiological investigations [5]. Serological procedures can be used for detecting subclinical infections of Babesia spp. However, these methods are not

Copyright: Bazmani, et al. Open Access. This article is distributed under the terms of the Creative Commons Attribution 4.0 International License (http://creativecommons.org/licenses/ by/4.0/), which permits unrestricted use, distribution, and reproduction in any medium, provided you give appropriate credit to the original author(s) and the source, provide a link to the Creative Commons license, and indicate if changes were made. The Creative Commons Public Domain Dedication waiver (http:// creativecommons.org/publicdomain/zero/1.0/) applies to the data made available in this article, unless otherwise stated. specific due to false positive and negative results and cross-reactivity with other species of Babesia [6].

The high sensitivity and specificity of molecular methods provide an accurate technique for differentiation of Babesia infections [7,8]. The prevalence of ovine babesiosis in Iran has been studied based on microscopic, serologic, and molecular approaches [1,9-12]. In the most of studies in Iran, $B$. ovis has been reported as the main causative agent of ovine babesiosis [1,11]. However, B. motasi was isolated in the blood samples of small ruminants in some studies in Iran [1,13]. Consequently, there is still an uncertainty regarding the causative agents of ovine babesiosis in small ruminants of Iran.

Therefore, the present study aimed to examine the presence of $18 \mathrm{~S}$ rRNA gene of both $B$. ovis and $B$. motasi in the blood samples of sheep and goats by the semi-nested polymerase chain reaction (PCR) technique.

\section{Materials and Methods}

\section{Ethical approval}

The study protocol was approved by the Ethical Committee of University of Tabriz (UT/D-0621).

\section{Study area}

This study was conducted in East Azerbaijan Province, Northwest of Iran during July 2015-September 2016 (Figure-1). The total area of this 


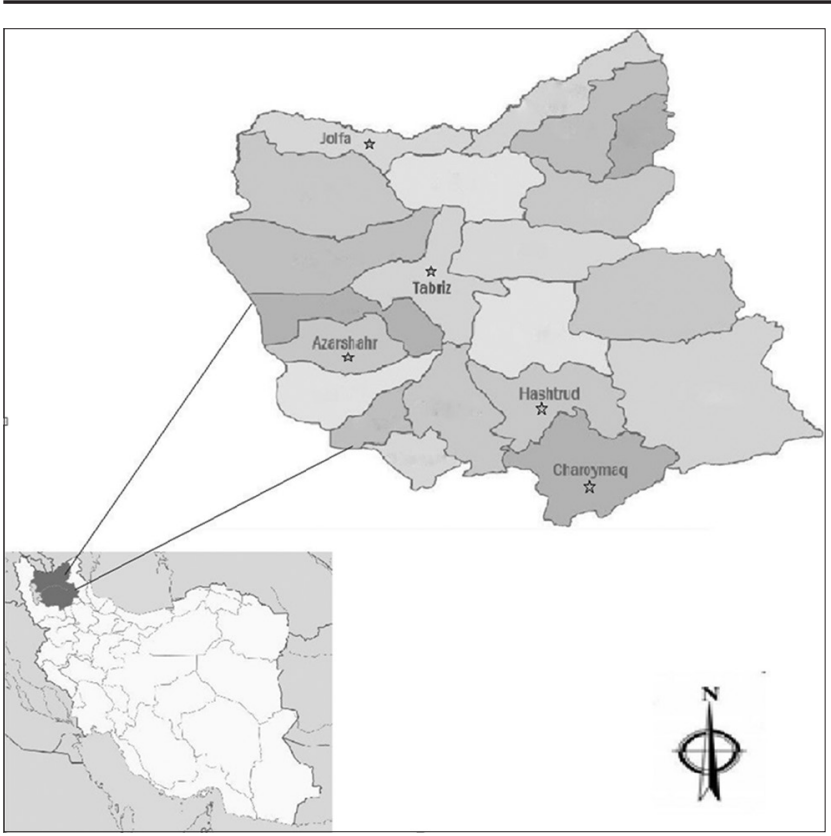

Figure-1: The map of East Azerbaijan Province of Iran. The blood samples were collected from areas which have been marked with a star.

province is approximately $45650 \mathrm{Km}^{2}$. The latitude and longitude are $36^{\circ} 54^{\prime}-39^{\circ} 26^{\prime} \mathrm{N}$ and $45^{\circ} 7^{\prime}-48^{\circ} 20^{\prime}$ $\mathrm{E}$, respectively. The average annual rainfall, relative humidity and temperature are $300 \mathrm{~mm}, 44-67 \%$ and $12.3^{\circ} \mathrm{C}$, respectively. The province has an arid to semi-arid climate. The sheep and goats farming is an important occupation in the rural areas of this province [14].

\section{Blood sampling}

In the present study, the following formula was used to determine the appropriate sample size for detection of disease prevalence:

$$
n=\frac{Z_{1-\alpha / 2}{ }^{2} p(1-p)}{d^{2}}
$$

Where; $\mathrm{n}=$ sample size, $Z_{1-\alpha / 2}=1.96$ for a confidence level of $95 \%, p=0.4$ based on previous studies in Iran, Error of margin $(d=0.08)$, therefore, $n=144$. A total of 166 blood samples were collected from the jugular vein of 123 sheep and 43 goats. Then, blood samples were filled in the $5 \mathrm{ml}$ vacutainer tubes containing EDTA and kept on ice until transferred to the laboratory. During sampling, information about age, gender, and species of animals was recorded through a questionnaire.

\section{DNA extraction}

The Genomic DNA of whole blood was extracted using a commercial kit (Pak Gene Yakhteh, Cat. No. PGEX2050) according to the manufacturer's instruction.

\section{PCR and semi-nested assays}

The PCR using an outer forward primer P1BF: 5'-CACAGGGAGGTAGTGACAAG-3' [15] and a reverse primer P2B-R: 5'-CTAAGAATTTCAC CTCTGACAGT-3' [16] was performed to amplify a piece of 18S rRNA gene of Babesia and Theileria genera. The expected size of PCR products for $B$. ovis and B. motasi were 390 and 397 bp, respectively, which could not be differentiated in this stage. The PCR reaction was performed in a total volume of $20 \mu \mathrm{l}$ containing $10 \times$ PCR buffer $2 \mu \mathrm{l}, \mathrm{MgCl}_{2} 2.5 \mathrm{mM}$, dNTP $0.2 \mathrm{mM}$, outer primers $0.2 \mathrm{pM}$ of each (Bioneer Inc., Korea), Taq DNA polymerase 2U, template DNA $1.6 \mu \mathrm{l}$ using the Astec PC818 thermal cycler (Astec, Japan). The thermal condition of PCR in this stage was $5 \mathrm{~min}$ incubation at $94^{\circ} \mathrm{C}$ for initial denaturation, 35 cycles of $40 \mathrm{~s}$ at $94^{\circ} \mathrm{C}, 40 \mathrm{~s}$ at $60^{\circ} \mathrm{C}, 60 \mathrm{~s}$ at $72^{\circ} \mathrm{C}$ and a final extension at $72^{\circ} \mathrm{C}$ for $7 \mathrm{~min}$. The PCR products were electrophoresed on $1.5 \%$ agarose gel using Safe stain (CinnaGen Co., Iran).

The semi-nested PCR was performed on the primary PCR products to differentiate Babesia spp. Apair of primers, P3ov-F: 5'-GGCCTTTGCGTTACTTTGA-3' (forward, AY260178 NCBI) [15] and P2BR: 5'- CTAAGAATTTCACCTCTGACAGT-3' (reverse) [16] were used to amplify a $178 \mathrm{bp}$ fragment of the $18 \mathrm{~S}$ rRNA gene of $B$. ovis. In addition, P4mot-F: 5'-CGCGATTCCGTTATTGGAG-3' (forward, AY260179 NCBI) [5] and P2B-R: 5 ' - C TA A GAAT T T C A C C T C T G A C A G T-3' (reverse) [16] primers were used for amplification of a $207 \mathrm{bp}$ fragment of the 18S rRNA gene of the B. motasi. In each nested reaction $1.7 \mu$ of the primary PCR product was used as the template in a total volume of $14 \mu \mathrm{l}$ containing $10 \times$ PCR buffer $1.4 \mu \mathrm{l}, \mathrm{MgCl}_{2} 2.5 \mathrm{mM}$, dNTP $0.2 \mathrm{mM}$, inner primers $0.2 \mathrm{pM}$ of each (Bioneer Inc., Korea), Taq DNA polymerase 1.4 U. The thermal condition for this stage was the same as described above. Finally, gel electrophoresis was performed on the PCR products using $1.5 \%$ agarose gel containing $15 \mu \mathrm{l} / \mathrm{dl}$ Safe stain (CinnaGen Co., Iran).

The PCR products with the expected size of both outer and inner segments were sequenced in forward and reverse directions (ABI 3130xl Genetic Analyzer, USA; Pouya Gostar Zhen Co., Iran). The validity of the obtained sequences was confirmed using nBLAST program and alignment to the corresponding sequences available in the GenBank.

\section{Statistical analysis}

Statistical analysis was performed using SPSS software (version 21.0, SPSS Inc.). The Chi-square test was used to assess the relationship between parasitic infection (presence of B. ovis or B. motasi) and age $(<1$ year old and $>1$ year old), gender (female and male), and species of animals (sheep and goats). $\mathrm{p}<0.05$ was considered significant.

\section{Results}

The nBLAST analysis and comparison of the PCR products sequences with the $B$. ovis $18 \mathrm{~S}$ small subunit ribosomal RNA gene sequence (GenBank: AY533146.1) demonstrated that the sequences were identical to the GenBank report (Figure-2). 
As shown in Figure-3, the PCR with the primers (P1B-F/P2B-R) indicated that 19 (11.44\%) out of 166 blood samples were infected to Babesia and Theileria spp. of which 16 (9.64\%) and 3 (1.8\%) samples were related to sheep and goats, respectively.

Semi-nested PCR with specific primers of B. ovis (P3ov-F/P2B-R) showed that among $166 \mathrm{PCR}$ products of the first stage, $38(22.89 \%)$ samples were positive for B. ovis infection (Figure-4).

It can be seen from the data in Table- 1 that there was no significant difference in the infection rate of $B$. ovis between the animals less and more than 1-year-old.

Furthermore, no significant relationship was observed between the $B$. ovis infection and gender of animals.

The number of infected sheep $29(17.5 \%)$ to B. ovis was more than the infected goats $9(5.4 \%)$. However, no significant differences were found between the species of animals and the infection rate of B. ovis (Table-1).

In addition, semi-nested PCR with specific primers of B. motasi (P4mot-F/P2B-R) was accomplished on the PCR products, but none of these samples exhibited any visible band for $B$. motasi on agarose gel electrophoresis (Figure-5).
The number of positive and negative cases in PCR and semi-nested PCR for B. ovis are shown in Table-2. Out of 38 positive samples in the semi-nested PCR of B. ovis, only 13 samples were also positive in the first PCR. Due to the low parasitemia, the remaining positive samples of semi-nested PCR (25 samples) did not produce a visible band in the first PCR.

Interestingly, among 19 positive samples of the first PCR, 6 samples did not show a visible band both in the semi-nested PCR of B. ovis and B. motasi.

\section{Discussion}

The most common technique for diagnosing of clinical babesiosis in small ruminants is Giemsa staining [17]. However, this method does not have good sensitivity and specificity for detecting different species of Babesia. In addition, it is not an appropriate method for identifying of subclinical babesiosis in sheep and goats [8]. Molecular methods can be a good alternative for detection of these conditions. In this regard, the traditional PCR technique was frequently used in the epidemiologic studies of piroplasmosis in small ruminants $[7,8,11]$. However, this method cannot differentiate the various species of Babesia and Theileria; so, another molecular technique such as semi-nested PCR or restriction fragment length

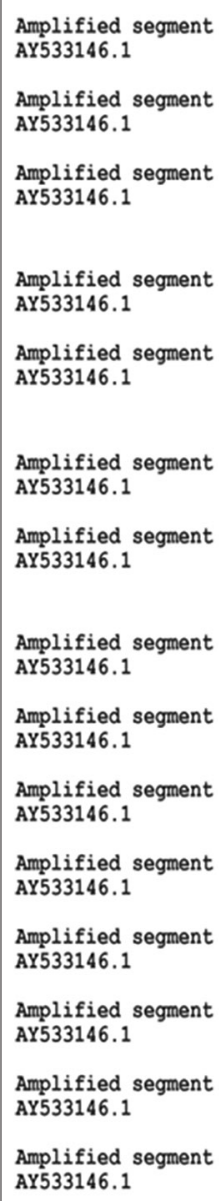

Figure-2: Alignment of amplified sequences to the Babesia ovis $18 \mathrm{~S}$ ribosomal RNA gene. Amplified target sequences are shown between two pairs of the corresponding primers. The amplified sequences were completely identical to the GenBank report (GenBank: AY260178.1). 
Table-1: The relationship between $B$. ovis infection and age, gender, and species of animals.

\begin{tabular}{|c|c|c|c|c|c|c|}
\hline \multirow[t]{3}{*}{ Results } & \multicolumn{6}{|l|}{ Variables } \\
\hline & \multicolumn{2}{|c|}{ Age } & \multicolumn{2}{|c|}{ Gender } & \multicolumn{2}{|c|}{ Species } \\
\hline & $<1$ year & $>1$ & Female & Male & Sheep & Goat \\
\hline Positive (\%) & $12(18.5)$ & $26(25.7)$ & $32(21.9)$ & $6(30)$ & $29(23.6)$ & $9(20.9)$ \\
\hline Negative (\%) & $53(81.5)$ & 75 (74.3) & $114(78.1)$ & $14(70)$ & $94(73.4)$ & $34(79.1)$ \\
\hline Total & 65 & 101 & 146 & 20 & 123 & 43 \\
\hline $\mathrm{p}$ value & \multicolumn{2}{|c|}{$p>0.05$} & \multicolumn{2}{|c|}{$p>0.05$} & \multicolumn{2}{|c|}{$p>0.05$} \\
\hline
\end{tabular}

Table-2: The number of positive and negative cases in PCR and semi-nested PCR of $B$. ovis.

\begin{tabular}{lcc}
\hline PCR Results & PCR+ & $\begin{array}{c}\text { Semi-nested } \\
\text { PCR of } \\
\text { B. ovis+ }\end{array}$ \\
\hline PCR+ & 19 & 13 \\
PCR- & 0 & 25 \\
Semi-nested PCR of B. ovis+ & 13 & 38 \\
Semi-nested PCR of B. ovis- & 6 & 0 \\
\hline
\end{tabular}

B. ovis=Babesia ovis, $\mathrm{PCR}=$ Polymerase chain reaction

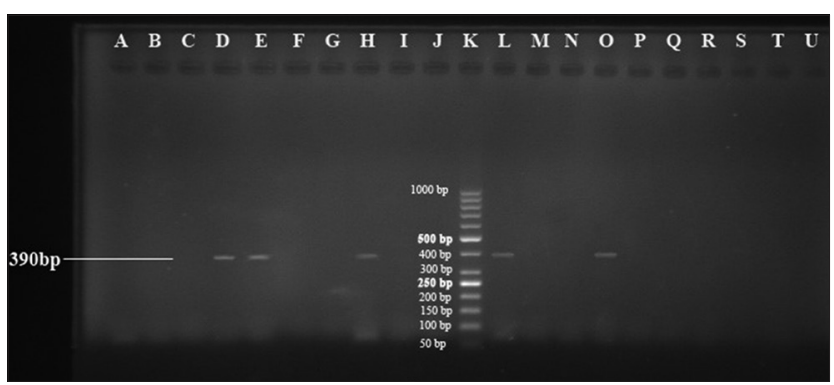

Figure-3: Primary polymerase chain reaction products were electrophoresed on $1.5 \%$ agarose gel to demonstrate a 390-442 bp fragment of 18S rRNA gene of Babesia and Theileria. Positive control: $L$; Negative control: $M$; Positive samples: D, E, H, O; Negative samples: A, B, C, F, G, I, J, $\mathrm{N}, \mathrm{P}, \mathrm{Q}, \mathrm{R}, \mathrm{S}, \mathrm{T}, \mathrm{U}$; Ladder 50 bp: $\mathrm{K}$.

polymorphism is needed for this purpose. Therefore, the present study was conducted to determine the frequency of $B$. ovis and B. motasi by semi-nested PCR in the blood samples of small ruminants in East Azerbaijan Province, Iran.

In the first PCR, 19 animals were found to be infected with Babesia or Theileria genus. Through the semi-nested PCR, the number of infection to $B$. ovis was 38 animals, although $B$. motasi infection was not detected in any of the examined animals. It is interesting to note that 6 positive samples of the first PCR did not show any visible band both in the semi-nested PCR for B. ovis and B. motasi. As a result, the latter positive samples may belong to another species of Babesia or Theileria.

In accordance with our results, most of the molecular studies demonstrated that $B$. ovis was the main causative agent of ovine babesiosis in Iran $[5,11]$. However, one case of B. motasi infection among 220 sheep and goats has been reported in a molecular study in Iran [13]. As well as molecular studies in the neighbor countries of Iran such as Turkey $[6,7,16,18]$ and Pakistan $[19,20]$ indicated that $B$. ovis was the

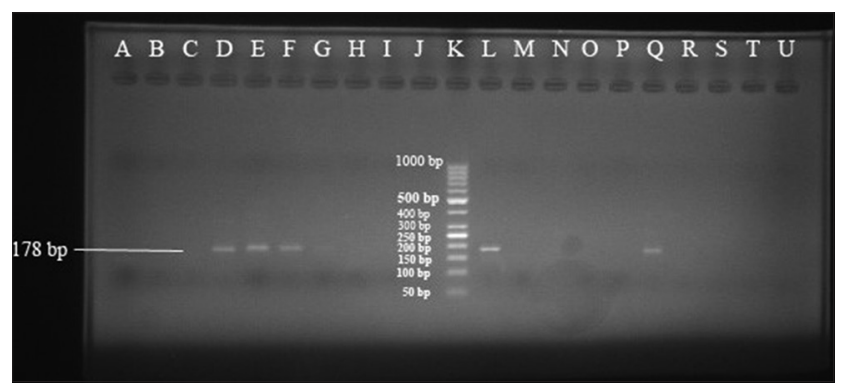

Figure-4: Semi-nested polymerase chain reaction products were electrophoresed on $1.5 \%$ agarose gel to demonstrate a 178 bp fragment of $18 \mathrm{~S}$ rRNA gene of Babesia ovis using specific primers. Positive control: $L$; Negative control: $M$; Positive samples: D, E, F, G, Q; Negative samples: A, B, C, $\mathrm{H}, \mathrm{I}, \mathrm{J}, \mathrm{N}, \mathrm{O}, \mathrm{P}, \mathrm{R}, \mathrm{S}, \mathrm{T}, \mathrm{U}$; Ladder $50 \mathrm{bp}$ : K.

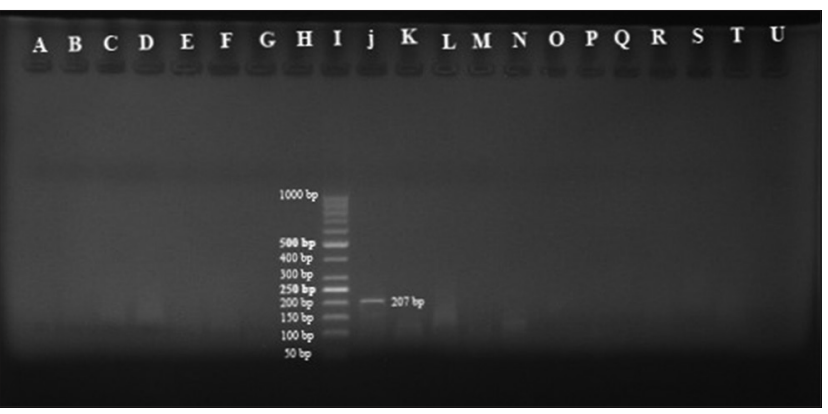

Figure-5: Semi-nested polymerase chain reaction products were electrophoresed on $1.5 \%$ agarose gel to demonstrate a 207 bp fragment of 18S rRNA gene of Babesia motasi using specific primers. Positive control: $\mathrm{J}$; Negative control: K; Negative samples: A, B, C, D, E, F, G, H, L, M, N, O, P, $\mathrm{Q}, \mathrm{R}, \mathrm{S}, \mathrm{T}, \mathrm{U}$; Ladder $50 \mathrm{bp}: \mathrm{I}$.

main causative agent of ovine babesiosis which were in agreement with our results.

The current study found that the infection rate of $B$. ovis among small ruminants, sheep, and goats was $22.89 \%, 23.6 \%$, and $20.9 \%$, respectively. Several studies have been conducted in different regions of Iran to detect the frequency of Babesia infection among sheep and goat populations. For example, in a biomorphometrical and molecular study, the frequency of $B$. ovis among 154 sheep was $5.85 \%$ [5]. Furthermore, the infection rate of $B$. ovis in the small ruminants of East Azerbaijan, West Azerbaijan, and Razavi Khorasan provinces was $14 \%, 16.7 \%$, and $0.99 \%$, respectively $[8,21,22]$. In accordance to our findings, a systematic review and meta-analysis on the status of babesiosis in Iran demonstrated that the prevalence of babesiosis in sheep and goats was $21.8 \%$ 
and $10.4 \%$, respectively [12]. Although it is difficult to compare the results of this and prior studies due to different sample size and various techniques used to identify $B$. ovis infection, the previous investigations indicate that ovine babesiosis is endemic in different regions of Iran.

The results of this study indicate that the number of infected animals with more than 1-year-old was greater than the animals with $<1$ year old. However, there was no significant difference between age and $B$. ovis infection. The present findings seem to be consistent with other researches which found no significant relationship between the age of the animal and infection with $B$. ovis $[4,6,19]$. In addition, no significant association was observed between the $B$. ovis infection and gender of animals. This finding is in agreement with the findings of Aktaş et al. [6], which showed that there was no significant relationship between gender and $B$. ovis infection. However, in contrast to our finding, Iqbal et al. [19] exhibited that the association between gender and $B$. ovis infection was statistically significant. A possible explanation for this might be that due to the increase of infected ticks in warm season in this area, all age groups and gender of sheep and goats are constantly subjected to tick bites; so, the state of enzootic stability has been established, as described by others $[6,17,23]$.

Results of the present study showed that the number of infected sheep more than infected goats. However, no significant difference was found between the species of animals and infection to $B$. ovis. In contrast to our results, some studies demonstrated that the susceptibility of sheep to $B$. ovis infection were more than goats $[4,8,19]$. It has been described that in the field condition, sheep were more susceptible to clinical disease caused by B. ovis infection than goats [24].

\section{Conclusion}

The present study set out to determine the prevalence of Babesia infection in sheep and goats in Northwest of Iran by the semi-nested PCR technique. The study has shown that the semi-nested PCR is a precise method for differentiation of Babesia spp. The results of this investigation indicate that Babesia infection is highly prevalent (22.89\%) among sheep and goat populations in East Azerbaijan Province, Northwest of Iran. In this study, we found no evidence for $B$. motasi infection. Therefore, it seems that $B$. ovis is the only species caused ovine Babesiosis in this region. The current study did not evaluate the tick vectors of ovine Babesiosis. Hence, further investigations on the tick vectors of babesiosis are needed to provide better control strategies for the disease.

\section{Authors' Contributions}

AB planned and carried out the study, AA carried out the study. AI planned, designed, and supervised the experiment. HA participated in the collecting data, analysis, and interpretation of data and drafting of the manuscript. All authors read and approved the final manuscript.

\section{Acknowledgments}

This study was conducted for an MSc thesis of Veterinary Parasitology (No. D-43-7541) and the financial supply was provided by the Faculty of Veterinary Medicine, University of Tabriz, Iran. The authors would like to appreciate the support and thank all of the field veterinarians for their collaboration.

\section{Competing Interests}

The authors declare that they have no competing interests.

\section{References}

1. Razmi, G., Naghibi, A., Aslani, M., Dastjerdi, K. and Hossieni, H. (2003) An epidemiological study on Babesia infection in small ruminants in Mashhad suburb, Khorasan province, Iran. Small Rumin. Res., 50: 39-44.

2. Uilenberg, G. (2006) Babesia-a historical overview. Vet. Parasitol., 138: 3-10.

3. Schnittger, L., Rodriguez, A.E., Florin-Christensen, M. and Morrison, D.A. (2012) Babesia: A world emerging. Infect. Genet. Evol., 12: 1788-1809.

4. Theodoropoulos, G., Gazouli, A., Ikonomopoulos, J.A., Kantzoura, V. and Kominakis, A. (2006) Determination of prevalence and risk factors of infection with Babesia in small ruminants from Greece by polymerase chain reaction amplification. Vet. Parasitol., 135: 99-104.

5. Dehkordi, Z.S., Zakeri, S., Nabian, S., Bahonar, A., Ghasemi, F., Noorollahi, F. and Rahbari, S. (2010) Molecular and biomorphometrical identification of ovine babesiosis in Iran. Iran J. Parasitol., 5: 21-30.

6. Aktas, M., Altay, K. and Dumanli, N. (2007) Determination of prevalence and risk factors for infection with Babesia ovis in small ruminants from Turkey by polymerase chain reaction. Parasitol. Res., 100: 797-802.

7. Aktaş, M., Altay, K. and Dumanli, N. (2005) Development of a polymerase chain reaction method for diagnosis of Babesia ovis infection in sheep and goats. Vet. Parasitol., 133(4): 277-281.

8. Esmaeilnejad, B., Tavassoli, M., Asri-Rezaei, S., Dalir-Naghadeh, B., Mardani, K., Golabi, M., Arjmand, J., Kazemnia, A. and Jalilzadeh, G. (2015) Determination of prevalence and risk factors of infection with Babesia ovis in small ruminants from west azerbaijan province, Iran by polymerase chain reaction. J. Arthropod Borne Dis., 9: 246-252.

9. Hashemzadeh, F.H., Nabavi, L., Seyfiabad, S.M., Rahbari, S. and Azizi, F. (2006) Development of an ELISA technique for the detection of Babesia ovis and serological survey of the parasite in Khouzestan province, Southern Iran. Iran J. Vet. Res., 7: 53-58.

10. Shayan, P., Hooshmand, E., Nabian, S. and Rahbari, S. (2008) Biometrical and genetical characterization of large Babesia ovis in Iran. Parasitol. Res., 103: 217-221.

11. Ranjbar-Bahadori, S., Eckert, B., Omidian, Z., Shirazi, N.S. and Shayan, P. (2012) Babesia ovis as the main causative agent of sheep babesiosis in Iran. Parasitol. Res., 110: 1531-1536.

12. Haghi, M.M., Etemadifar, F., Fakhar, M., Teshnizi, S.H., Soosaraei, M., Shokri, A., Hajihasani, A. and Mashhadi, H. (2017) Status of babesiosis among domestic herbivores in Iran: A systematic review and meta-analysis. Parasitol. Res., 116: 1101-1109.

13. Motavalli, H.S.M., Fakar, M., Sharif, M., Paghe, A., Sharbatkhori, M., Tavakoli, R. and Gholami, S. (2013) Molecular identification of ovine Babesia spp. in north of Iran. Res. Mol. Med., 1: 36-40. 
14. Abai, M., Azari-Hamidian, S., Ladonni, H., Hakimi, M., Mashhadi-Esmail, K., Sheikhzadeh, K., Kousha, A. and Vatandoost, H. (2007) Fauna and checklist of mosquitoes (Diptera: Culicidae) of east azerbaijan province, northwestern Iran. J. Arthropod Borne Dis., 1: 27-33.

15. Shayan, P. and Rahbari, S. (2005) Simultaeus differentiation between Theileria spp. and Babesia spp. on stained blood smear using PCR. Parasitol. Res., 97: 281-286.

16. Aydin, M.F., Aktas, M. and Dumanli, N. (2013) Molecular identification of Theileria and Babesia in sheep and goats in the black sea region in Turkey. Parasitol. Res., 112: $2817-2824$

17. Razmi, GR., Naghibi, A., Aslani, M., Fathivand, M. and Dastjerdi, K. (2002) An epidemiological study on ovine babesiosis in the Mashhad suburb area, province of Khorasan, Iran. Vet. Parasitol., 108: 109-115.

18. Altay, K., Dumanli, N. and Aktas, M. (2007) Molecular identification, genetic diversity and distribution of Theileria and Babesia species infecting small ruminants. Vet. Parasitol., 147: 161-165.

19. Iqbal, F., Fatima, M., Shahnawaz, S., Naeem, M., Shaikh, R., Ali, M., Shaikh, A. and Aktas, M. (2011) A study on the determination of risk factors associated with babesiosis and prevalence of Babesia sp., by PCR amplification, in small ruminants from Southern Punjab (Pakistan).
Parasite, 18: 229-234.

20. Shahzad, W., Noor, H., Ahmad, M.U.D., Munir, R., Saghar, M.S., Mushtaq, M.H., Ahmad, N., Akbar, G. and Mehmood, F. (2013) Prevalence and molecular diagnosis of Babesia ovis and Theileria ovis in lohi sheep at livestock experiment station (LES), Bahadurnagar, Okara, Pakistan. Iran J. Parasitol., 8: 570-578.

21. Shahbazi, A., Fallah, E., Mamagani, A.J., Khanmohammadi, M., Nematollahi, A. and Bazmani, A. (2013) Identification of Babesia species in sheep isolated from villages of East Azerbaijan by Semi-nested PCR. $J$. Pure Appl. Microbiol., 7: 201-205.

22. Razmi, G., Pourhosseini, M., Yaghfouri, S., Rashidi, A. and Seidabadi, M. (2013) Molecular detection of Theileria spp. and Babesia spp. in sheep and ixodid ticks from the northeast of Iran. J. Parasitol., 99: 77-81.

23. Rjeibi, M.R., Gharbi, M., Mhadhbi, M., Mabrouk, W., Ayari, B., Nasfi, I., Jedidi, M., Sassi, L., Rekik, M. and Darghouth, M.A. (2014) Prevalence of piroplasms in small ruminants in North-West Tunisia and the first genetic characterisation of Babesia ovis in Africa. Parasite, 21: 23.

24. Friedhoff, K.T. (1997) Tick-borne diseases of sheep and goats caused by Babesia, Theileria or Anaplasma spp. Parassitologia, 39: 99-109. 